\title{
A hitherto unrecognized source of low-energy electrons in water
}

\author{
Melanie Mucke', Markus Braune ${ }^{2}$, Silko Barth ${ }^{1}$, Marko Förstel ${ }^{1,3}$, Toralf Lischke ${ }^{1}$, Volker Ulrich ${ }^{1}$, \\ Tiberiu Arion', Uwe Becker ${ }^{2}$, Alex Bradshaw ${ }^{1,2}$ and Uwe Hergenhahn ${ }^{1,4 \star}$
}

Low-energy electrons are the most abundant product of ionizing radiation in condensed matter. The origin of these electrons is most commonly understood to be secondary electrons ${ }^{1}$ ionized from core or valence levels by incident radiation and slowed by multiple inelastic scattering events. Here, we investigate the production of low-energy electrons in amorphous medium-sized water clusters, which simulate water molecules in an aqueous environment. We identify a hitherto unrecognized extra source of low-energy electrons produced by a non-local autoionization process called intermolecular coulombic decay ${ }^{2}$ (ICD). The unequivocal signature of this process is observed in coincidence measurements of lowenergy electrons and photoelectrons generated from innervalence states with vacuum-ultraviolet light. As ICD is expected to take place universally in weakly bound aggregates containing light atoms between carbon and neon in the periodic table $e^{2,3}$, these results could have implications for our understanding of ionization damage in living tissues.

Electronic vacancy states can be produced in matter by ionizing radiation, such as X-ray photons or fast charged particles. When a state with a high electronic excitation energy has been produced by impact of such particles, electron correlation can cause the ejection of electrons. Auger decay is the best known representative of this class of secondary processes that is more generally termed autoionization. In the case of Auger decay, a vacancy in an inner shell is filled by a transition involving electrons of lower binding energy at the same atom. For extended systems of weakly bound constituents, such as van-der-Waals or hydrogen-bonded clusters, Cederbaum et al. ${ }^{2}$ have found that a different mechanism pertains. They predicted that in such systems electron correlation can effect an Auger-like autoionization transition in which valence electrons on neighbouring sites participate. In other words, the mechanism is a concerted transition in which a single hole in an inner shell is replaced by two vacancies in the outer valence shells of two adjacent molecules, and a free electron. This decay channel was termed intermolecular (interatomic, in the case of atomic clusters) coulombic decay and was subsequently observed in rare-gas clusters ${ }^{4-7}$. The process is shown schematically in Fig. 1. A resonant variant of ICD, which may take place after photoexcitation into an unoccupied orbital, has also been discussed ${ }^{7-9}$. Here, we consider ICD of inner-valence vacancy states, for which case the ejected electrons have a low kinetic energy.

On the basis of energetic considerations, ICD can take place whenever the binding energy of the ionized state lies above the double-ionization threshold of the corresponding cluster or liquid. This prerequisite for ICD is fulfilled in hydrogen-bonded a

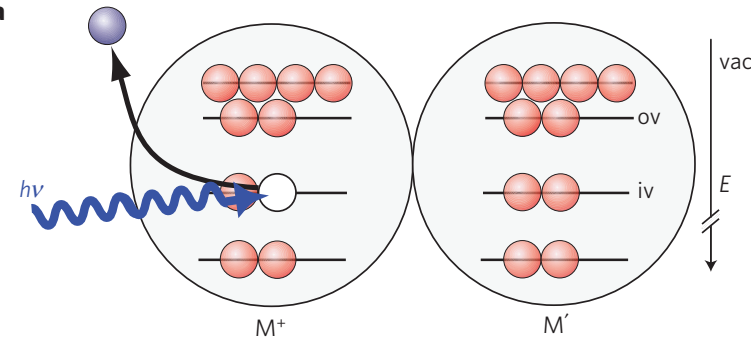

b

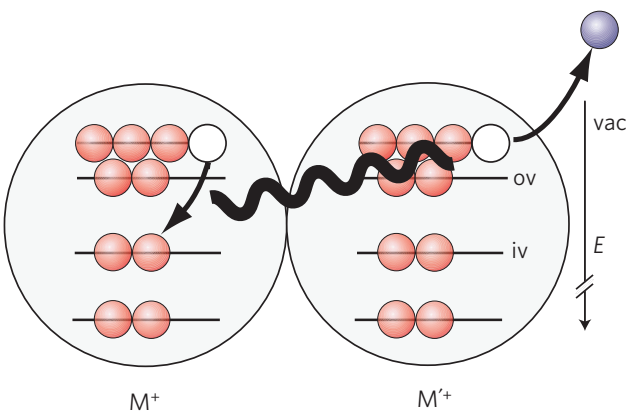

Figure 1 | Schematic of ICD of an inner-valence vacancy in a hydrogen-bonded network of water molecules. $\mathbf{a}, \mathbf{b}$, This interatomic autoionization process takes place when an inner-valence (iv) vacancy created by (photo)ionization on molecule $M(\mathbf{a})$ is filled by an electron from an outer-valence (ov) orbital of the same molecule, while another outer-valence electron is emitted from the nearest-neighbour molecule $\mathrm{M}^{\prime}$ (see b). 'vac' designates the vacuum level or ionization potential, in an isolated system.

systems ${ }^{2,10}$, but so far the process has not been seen. Calculations of the energy spectrum of electrons ejected by ICD of small water clusters give a hint as to why it has escaped observation: a broad, rather unstructured distribution of energies is expected, which peaks at $0 \mathrm{eV}$ (ref. 10). If we consider an experiment with a conventional electron energy analyser on a bulk or liquid sample, an electron spectrum with this shape can hardly be distinguished from the 'universal curve'l for secondary electrons (Fig. 2). In this respect our work differs from earlier experiments, which were either restricted to dimers ${ }^{5-7}$, or dealt with simpler cases where an ICD feature appears from simple electron kinetic energy spectra ${ }^{4,8,9}$. Producing primary electrons of a well-defined energy by photoionization and detecting them in coincidence with the ICD electron has enabled us to overcome the aforementioned problem. Here, we demonstrate that ICD follows the photoionization of medium-sized water

\footnotetext{
${ }^{1}$ Max-Planck-Institut für Plasmaphysik, EURATOM Association, Boltzmannstr. 2, 85748 Garching, Germany, ${ }^{2}$ Fritz-Haber-Institut der Max-Planck-Gesellschaft, Faradayweg 4-6, 14195 Berlin, Germany, ${ }^{3}$ Max-Planck-Institut für Kernphysik, Saupfercheckweg 1, 69117 Heidelberg, Germany, ${ }^{4}$ Helmholtz-Zentrum Berlin, Albert-Einstein-Str. 15, 12489 Berlin, Germany. ${ }^{\star}$ e-mail: uwe.hergenhahn@ipp.mpg.de.
} 


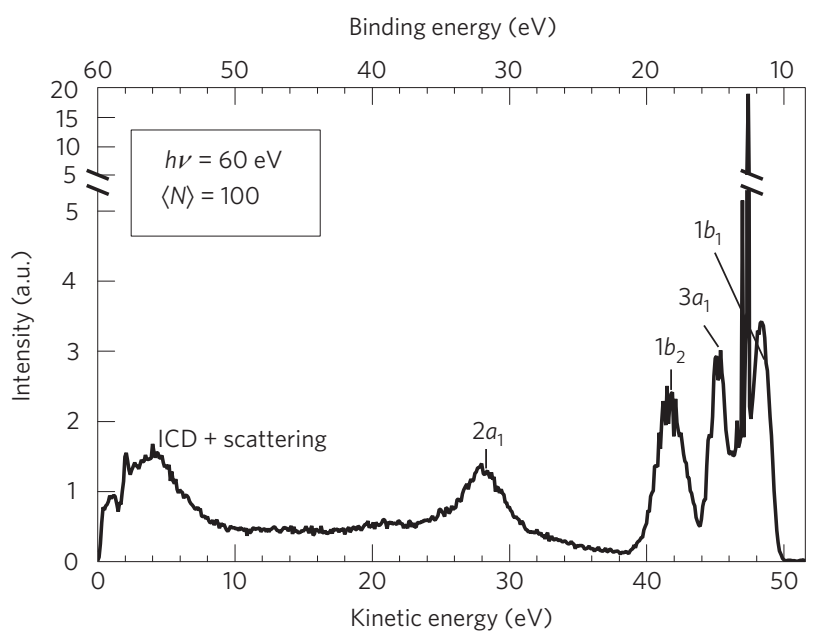

Figure 2 | Full photoelectron spectrum of water clusters recorded with a conventional electron spectrometer ${ }^{\mathbf{1 2}}$. Here, we describe autoionization of the $2 a_{1}$ vacancy states by emission of low-energy electrons, which contribute to the intensity below $10 \mathrm{eV}$. Formerly this was thought to be composed solely of electrons that have been multiply inelastically scattered. The sharp lines in the outer-valence region are due to the vibrational fine structure of the superimposed spectrum of water monomers.

clusters and show that-above the corresponding photoionization threshold-ICD electrons make an important contribution to the low-kinetic-energy spectrum.

In our experiment, a jet of water clusters with a mean size $\langle N\rangle$ of 40 and 200 was used. Such clusters are believed to form amorphous structures, which resemble the hydrogen-bonded network of liquid water rather than that of crystalline ice ${ }^{11}$. Inner-valence vacancies were produced by photoionization using synchrotron radiation. The complete non-coincident photoelectron spectrum of water clusters, recorded in a separate experiment with a hemispherical electron energy analyser ${ }^{12}$, is shown in Fig. 2. It is similar to that of liquid water recorded previously ${ }^{13}$. At the excitation energy range covered in this work the ICD process can take place for the $2 \mathrm{a}_{1}$ inner-valence vacancy states, which are mainly derived from the $\mathrm{O} 2 s$ orbitals.

As described above, it is necessary to record simultaneously both electrons involved to show that ICD actually occurs. For this coincidence experiment we have used a so-called magnetic bottle time-of-flight electron spectrometer, which is well suited for this purpose owing to its large acceptance angle and good transmission for electrons down to very low kinetic energies (see the Methods section). In the lower right panel of Fig. 3, we show the yield of electron pairs $\left(e_{1}, e_{2}\right)$ recorded in a coincidence experiment at a photon energy of $45 \mathrm{eV}$. The kinetic energy of the higher-energy electron $e_{1}$ increases along the vertical axis; that of the lower-energy electron $e_{2}$ along the horizontal axis. Correlating the energies along the $e_{1}$ axis with Fig. 2, we can see which parts of the spectrum are characterized by strong emission of very low-energy electrons. We observe in particular the feature between the two red lines in the figure: these pairs consist of an $e_{1}$ electron with a kinetic energy corresponding to inner-valence photoionization and an $e_{2}$ electron, with a very low kinetic energy. The energy spectrum of all $e_{2}$ electrons detected in coincidence with an inner-valence electron $e_{1}$, within the energy interval marked by the red bars in Fig. 3, is shown in the top panel. It consists of a convolution of the singly ionized states with binding energies in the range of $28-35 \mathrm{eV}$ (ref. 10) with all available two-hole final states. The resulting intensity profile decreases from zero towards higher kinetic energy and has similarly been found in calculations ${ }^{10}$ for
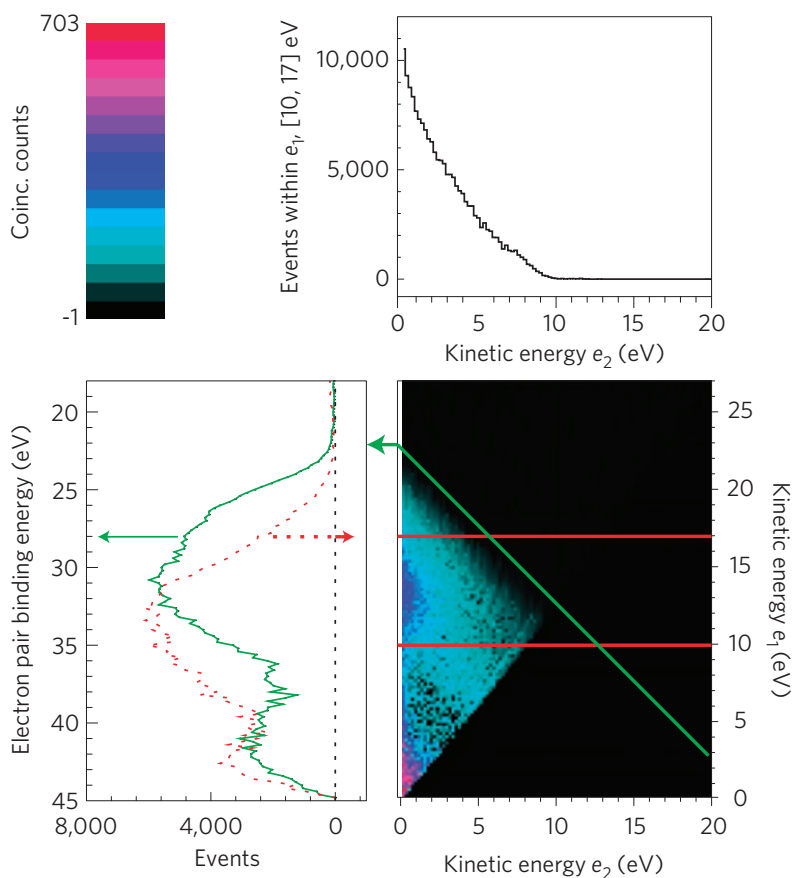

Figure 3 | Coincidence spectrum of primary and secondary electrons produced by ionization of $\langle N\rangle=\mathbf{4 0}$ water clusters with photons of $\mathbf{4 5} \mathrm{eV}$ energy. Lower right panel: intensity of electron pairs with kinetic energies as recorded on the right-hand vertical axis (fast electron, $e_{1}$ ) and on the horizontal axis (slow electron, $e_{2}$ ) shown as a colour-coded map. The region between the two red bars is assigned primarily to photoelectron-ICD electron pairs. The energy spectrum of ICD electrons, integrated over the above-mentioned range of photoelectron energies, is shown in the top panel. The photoelectron spectrum as a function of kinetic energy, integrated over all energies of the $e_{2}$ electron, is shown in the left panel (red dotted line, referring to the right-hand vertical axis; see Supplementary Information). In this panel we also show the intensity of electron pairs as a function of pair binding energy, or two-hole final state energy (green trace, referring to the left-hand vertical axis).

water clusters up to the tetramer. In analogy to earlier experiments on rare-gas clusters ${ }^{4-7}$, we identify this autoionization process with intermolecular coulombic decay. As expected, the respective feature is not observed for a beam consisting purely of water monomers (see Supplementary Fig. S1). We can further confirm the assignment by following the photon energy dependence: the $e_{1}$ energy of the ICD feature should vary linearly with photon energy, whereas the $e_{2}$ energy remains constant. Exactly this behaviour is observed in Fig. 4, which shows the results of coincidence experiments at photon energies of 45, 60 and $80 \mathrm{eV}$.

The doubly charged final states, which are populated by the ICD process, can be inferred from a plot of the electron coincidence intensities as a function of final two-hole-state energy (Fig. 3, green curve). This curve is obtained by integrating along lines of constant total energy, which are diagonals in the main panel parallel to the green line. This line itself marks the minimum final-state energy expected, namely $22.1 \mathrm{eV}$ corresponding to twice the highest occupied molecular orbital ionization potential of a large water cluster ${ }^{12}$. As the final-state spectrum is composed of numerous combinations of outer-valence single vacancies ${ }^{10}$, and is further broadened by summation over different cluster geometries, a broadened double-ionization spectrum between 23 and $36 \mathrm{eV}$ is observed. These final-state energies correspond to the production of two outer-valence vacancies, mostly as a result of ICD. The intensity of the electron coincidences (green curve) at higher binding energies is partly due to direct photo-double-ionization of uncondensed 

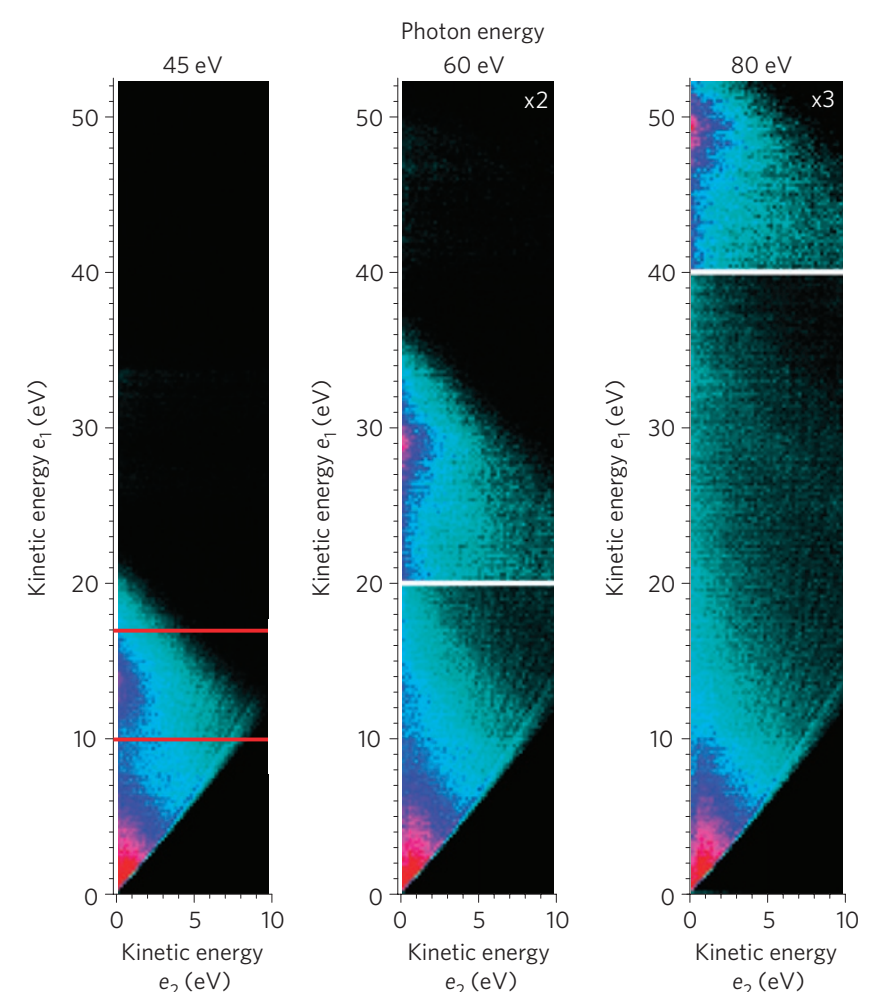

Figure $4 \mid$ Coincidence electron spectra of $\langle\mathbf{N}\rangle=\mathbf{4 0}$ water clusters recorded at three different photon energies. A change of scale in the middle and right-hand panels has been introduced for better visibility. Whereas the energy of $e_{1}$ increases with photon energy, the energy spectrum of $e_{2}$ is virtually unchanged. This corroborates our interpretation as a two-step process. An intensity of electron pairs with kinetic energies both below approximately $5 \mathrm{eV}$ is always observed, and is believed to originate from events involving an unobserved third particle, or from the spectrometer.

molecules in the jet, which can result in the creation of molecular dicationic states with up to $47 \mathrm{eV}$ binding energy ${ }^{14}$. Intra cluster scattering processes also make a minor contribution to the electronpair intensities in Figs 3 and 4. Further details in connection with these figures, such as subtraction of random coincidences, are discussed in the Methods section and Supplementary Information.

The above analysis was made under the assumption that no nuclear relaxation occurs during ICD. The timescale of this process has been investigated experimentally $y^{5,15}$ and by calculations ${ }^{3,16}$ for a number of systems, including water clusters ${ }^{10}$. Transition times in the femtosecond range have been found. This is even faster than the proton rearrangement associated with the ionization of water ${ }^{17}$. Autoionization through ICD therefore will dominate over alternative relaxation channels, such as fluorescence or nuclear rearrangement. For Ne clusters, 100\% efficiency of ICD for the relaxation of $2 s$ vacancies has been demonstrated ${ }^{18}$.

We will now briefly comment on the possible impact of these results. It is well known that even low doses of ionizing radiation can lead to cell damage in living matter, and that such events primarily are caused by double-strand breaks in the DNA involved ${ }^{19,20}$. The mechanisms that lead from absorption of the radiation energy to double-strand breaks are far from understood. One possible process is the chemical attack by $\mathrm{OH}$ radicals resulting from the radiolysis of water ${ }^{19,20}$. Recently the potential importance of low-kinetic-energy electrons has been recognized $^{21,22}$, as it was found that they can efficiently induce DNA strand breaks by dissociative attachment ${ }^{23-25}$. These results have now been confirmed in humid air, thus resembling more closely conditions in the cell ${ }^{26}$. As electron attachment can be promoted by so-called shape resonances, there is practically no lower limit for the electron energy at which an interaction with DNA ceases to occur ${ }^{27,28}$. Intermolecular coulombic decay provides a production mechanism for low-kinetic-energy electrons not considered previously. For primary ionization with large impact energy, it differs from other mechanisms, such as multiple inelastic scattering of high-kinetic-energy photoelectrons, by the fact that the ICD electron is produced immediately at the site of ionization. Quantitatively, for vacuum-ultraviolet photon energies, an upper limit for the amount of slow electrons expected from ICD is given by the relative inner-valence photoionization cross-section, which is $18 \%$ for $h v=80 \mathrm{eV}$ (ref. 13) and could be higher near threshold. For higher primary energies, ICD can also occur as part of a cascade process following normal Auger decay ${ }^{6}$. Although it is difficult to assess the number of resulting ICD electrons as a fraction of the 'true' secondaries (largely because of the problem of quantifying the latter), their contribution is clearly important.

Another aspect contributing to the relevance of ICD is the production of two positively charged ions in its final state. For our system, their nuclear Coulomb repulsion energy, calculated at the original water-water bond length ${ }^{10}$, is around $4.9 \mathrm{eV}$. This will lead to a Coulomb explosion in a small system, and to structural changes at the site of ICD in larger aggregates.

Finally, we see important perspectives in the study of ICD in inhomogeneous systems such as, for example, chemical systems; DNA itself might participate in such a process.

Note added in proof: The Coulomb explosion of water dimers after ICD has very recently been independently observed ${ }^{29}$.

\section{Methods}

Cluster production. Clusters were produced by supersonic expansion of water vapour from a heated reservoir inside the vacuum chamber. Data specific to our instrument are: conical copper nozzle, diameter $80 \mu \mathrm{m}$, length $1.1 \mathrm{~mm}$, half opening angle $15^{\circ}$. The expansion chamber and main chamber are pumped by two $2101 \mathrm{~s}^{-1}$ and one $1,000 \mathrm{ls}^{-1}$ turbopumps, respectively, and are separated by a conical skimmer of $1 \mathrm{~mm}$ diameter. Background pressure in the main chamber was kept below $10^{-5} \mathrm{mbar}$. The cluster size was estimated from the empirical formula given in ref. 30. Cluster formation was monitored by recording the highest occupied molecular orbital photoelectron peaks of water monomers and clusters at $14 \mathrm{eV}$ photon energy ${ }^{12}$.

Electron detection. The magnetic bottle spectrometer we have used features an adaptation of an earlier design ${ }^{31}$ to experiments with the bunch period of BESSY II, which is $800 \mathrm{~ns}$. We have therefore shortened the drift tube to $60 \mathrm{~cm}$. The guiding field is produced by a coil of Kapton-insulated cable wound around the drift tube inside the vacuum. The anisotropic field near the interaction region is produced by a permanent magnet, generating a magnetic field strength of approximately $0.4 \mathrm{~T}$. Backscattering of electrons from the magnet surface is prevented by a mesh in front of the magnet to which a positive bias voltage was applied. Electrons with kinetic energies down to $100 \mathrm{meV}$ were detected without loss of transmission, which was verified by recording He $1 s$ photoelectron spectra with photon energies down to the ionization threshold. A small accelerating field $(1.2 \mathrm{~V})$ along the interaction region was used to achieve a time-of-flight of the slowest electrons below $800 \mathrm{~ns}$.

The complete non-coincident valence photoelectron spectrum shown in Fig. 2 was recorded in a separate experiment, which is described elsewhere ${ }^{12}$. Briefly, a conventional hemispherical analyser was used to record photoelectrons emitted under the magic angle with respect to the polarization direction of the light, and in the plane perpendicular to its propagation direction. A pass energy of $20 \mathrm{eV}$ was used. Although we have not quantified it, we expect that under these conditions the transmission function of the analyser leads to a decrease in the detection efficiency for electrons with kinetic energies below about $8 \mathrm{eV}$. A quantitative comparison of Fig. 2 to the low-kinetic-energy-electron spectra acquired with the magnetic bottle spectrometer is hampered by this problem.

Synchrotron radiation. Experiments were carried out at the undulator beamline UE 112-low energy-PGMa at the synchrotron radiation source BESSY II (Berlin, Germany), operating in single-bunch mode. The light polarization was chosen as horizontally linear, that is, perpendicular to the cluster beam and within the plane spanned by the photon beam and the central axis of the magnetic bottle spectrometer. 
Data acquisition and analysis. Event-based data acquisition was carried out with a multi-hit capable time-to-digital converter of $60 \mathrm{ps}$ bin width (GPTA) The electron flight times were transformed into kinetic energies according to calibration data obtained from $\mathrm{He} 1 s$ photoelectron spectra. The amount of random coincidences was estimated from electron pairs produced by two different, subsequent synchrotron radiation pulses, and was subtracted. In Fig. 3, additionally a background of coincident electron pairs of apparative origin was subtracted. This background consists mainly of electron pairs with a total kinetic energy below $10 \mathrm{eV}$, and was estimated from a spectrum of gaseous $\mathrm{He}$ recorded at $44.7 \mathrm{eV}$, which was scaled with the ratio of outer-valence photoelectron intensities. No such subtraction has been carried out in Fig. 4 to highlight the fact that the background in this energy region is essentially constant in shape.

Typical acquisition times were 1,000 s per panel. In this period, for Fig. 3 about 11 million total events were registered, which could be condensed to 366,000 true coincidence events, after background subtraction. These figures are typical for the other spectra as well. The coincidence electron spectra in the three panels of Fig. 4 were normalized to equal maximum intensity.

Received 23 June 2009; accepted 25 November 2009; published online 10 January 2010

\section{References}

1. Seiler, H. Secondary electron emission in the scanning electron microscope J. Appl. Phys. 54, R1-R18 (1983).

2. Cederbaum, L. S., Zobeley, J. \& Tarantelli, F. Giant intermolecular decay and fragmentation of clusters. Phys. Rev. Lett. 79, 4778-4781 (1997).

3. Santra, R. \& Cederbaum, L. S. Non-Hermitian electronic theory and applications to clusters. Phys. Rep. 368, 1-117 (2002).

4. Marburger, S., Kugeler, O., Hergenhahn, U. \& Möller, T. Experimental evidence for interatomic coulombic decay in Ne clusters. Phys. Rev. Lett. 90, 203401 (2003).

5. Jahnke, T. et al. Experimental observation of interatomic coulombic decay in neon dimers. Phys. Rev. Lett. 93, 163401 (2004).

6. Morishita, Y. et al. Experimental evidence of interatomic coulombic decay from the Auger final states in argon dimers. Phys. Rev. Lett. 96, 243402 (2006).

7. Aoto, T. et al. Properties of resonant interatomic coulombic decay in Ne dimers. Phys. Rev. Lett. 97, 243401 (2006).

8. Barth, S. et al. Observation of resonant interatomic coulombic decay in $\mathrm{Ne}$ clusters. J. Chem. Phys. 122, 241102 (2005)

9. Aziz, E. F., Ottosson, N., Faubel, M., Hertel, I. V. \& Winter, B. Interaction between liquid water and hydroxide revealed by core-hole de-excitation. Nature 455, 89-91 (2008).

10. Müller, I. B. \& Cederbaum, L. S. Ionization and double ionization of small water clusters. J. Chem. Phys. 125, 204305 (2006).

11. Buch, V., Bauerecker, S., Devlin, J. P., Buck, U. \& Kazimirski, J. K. Solid water clusters in the size range of tens-thousands of $\mathrm{H}_{2} \mathrm{O}$ : A combined computational/spectroscopic outlook. Int. Rev. Phys. Chem. 23, 375-433 (2004).

12. Barth, S. et al. Valence ionization of water clusters: From isolated molecules to bulk. J. Phys. Chem. A 113, 13519-13527 (2009).

13. Winter, B. et al. Full valence band photoemission from liquid water using EUV synchrotron radiation. J. Phys. Chem. A 108, 2625-2632 (2004).

14. Eland, J. H. D. Double photoionisation spectra of methane, ammonia and water. Chem. Phys. 323, 391-396 (2006).

15. Öhrwall, G. et al. Femtosecond interatomic coulombic decay in free neon clusters: Large lifetime differences between surface and bulk. Phys. Rev. Lett. 93, 173401 (2004)
16. Kuleff, A. I. \& Cederbaum, L. S. Tracing ultrafast interatomic electronic decay processes in real time and space. Phys. Rev. Lett. 98, 083201-083204 (2007).

17. Furuhama, A., Dupuis, M. \& Hirao, K. Reactions associated with ionization in water: A direct ab initio dynamics study of ionization in $\left(\mathrm{H}_{2} \mathrm{O}\right)_{17}$. J. Chem. Phys. 124, 164310 (2006)

18. Barth, S. et al. The efficiency of interatomic coulombic decay in Ne clusters. Chem. Phys. 329, 246-250 (2006).

19. Ward, J. F. The complexity of DNA damage: Relevance to biological consequences. Int. J. Radiat. Biol. 66, 427-432 (1994).

20. O'Neill, P. in Radiation Chemistry: Present Status and Future Trends (eds Jonah, C. D. \& Rao, B. S. M.) 585-622 (Elsevier, 2001).

21. Folkard, M. et al. Measurement of DNA damage by electrons with energies between 25 and $4000 \mathrm{eV}$. Int. J. Radiat. Biol. 64, 651-658 (1993).

22. Pimblott, S. M. \& LaVerne, J. A. Production of low-energy electrons by ionizing radiation. Radiat. Phys. Chem. 76, 1244-1247 (2007).

23. Michael, B. D. \& O'Neill, P. Molecular biology: A sting in the tail of electron tracks. Science 287, 1603-1604 (2000).

24. Boudaiffa, B., Cloutier, P., Hunting, D., Huels, M. A. \& Sanche, L. Resonant formation of DNA strand breaks by low-energy ( 3 to $20 \mathrm{eV}$ ) electrons. Science 287, 1658-1660 (2000)

25. Pan, X., Cloutier, P., Hunting, D. \& Sanche, L. Dissociative electron attachment to DNA. Phys. Rev. Lett. 90, 208102 (2003).

26. Brun, E., Cloutier, P., Sicard-Roselli, C., Fromm, M. \& Sanche, L. Damage induced to DNA by low-energy $(0-30 \mathrm{eV})$ electrons under vacuum and atmospheric conditions. J. Phys. Chem. B 113, 10008-10013 (2009).

27. Martin, F. et al. DNA strand breaks induced by $0-4 \mathrm{eV}$ electrons: The role of shape resonances. Phys. Rev. Lett. 93, 068101 (2004).

28. Bald, I., Kopyra, J., Dabkowska, I., Antonsson, E. \& Illenberger, E. Low energy electron-induced reactions in gas phase 1,2,3,5-tetra-O-acetyl-beta-D-ribofuranose: A model system for the behaviour of sugar in DNA. J. Chem. Phys. 126, 074308 (2007).

29. Jahnke, T. et al. Ultrafast energy transfer between water molecules. Nature Phys. doi:10.1038/nphys1498 (2010).

30. Bobbert, C., Schütte, S., Steinbach, C. \& Buck, U. Fragmentation and reliable size distributions of large ammonia and water clusters. Eur. Phys. J. D 19, 183-192 (2002).

31. Lablanquie, P. et al. Multielectron spectroscopy: Auger decays of the argon $2 \mathrm{p}$ hole. J. Electron Spectrosc. Relat. Phenom. 156-158, 51-57 (2007)

\section{Acknowledgements}

U.H. would like to acknowledge useful discussions with B. Winter. Financial support by the Deutsche Forschungsgemeinschaft, the Fonds der Chemischen Industrie and the Advanced Study Group of the Max-Planck-Society is gratefully acknowledged.

\section{Author contributions}

U.H. conceived the experiment, S.B. and V.U. constructed the cluster source, M.B. and U.B. constructed the spectrometer, M.M., M.B., S.B., M.F., T.L. and U.H. carried out the experiment and M.M. and U.H. analysed the data; all authors discussed the results and contributed to writing of the manuscript.

\section{Additional information}

The authors declare no competing financial interests. Supplementary information accompanies this paper on www.nature.com/naturephysics. Reprints and permissions information is available online at http://npg.nature.com/reprintsandpermissions. Correspondence and requests for materials should be addressed to U.H. 